\title{
THE SPATIAL STRUCTURE OF THE WMAP/PLANCK HAZE
}

\author{
Yana Zhezher ${ }^{1,2, \star}$ \\ ${ }^{1}$ Institute for Nuclear Research, Russian Academy of Sciences, 117312, Moscow, Russia \\ ${ }^{2}$ Faculty of Physics, Lomonosov Moscow State University, 119991, Moscow, Russia
}

\begin{abstract}
It was recently proposed that the two phenomena, WMAP/Planck haze and Fermi bubbles may have a common origin. In the present paper we analyze the spatial structure of the haze using the Planck 2015 data. It is found that the spatial dimensions and locations of WMAP/Planck haze and Fermi bubbles are compatible within experimental uncertainties for symmetric approximation case, and WMAP/Planck haze appear to be more flattened in galactic latitude direction for the asymmetric approximation case. WMAP/Planck haze possesses north-south asymmetry, it is more intensive in the northern hemisphere. No substructures similar to Fermi bubbles cocoon are identified in Planck data.
\end{abstract}

\section{Introduction}

The Wilkinson Microwave Anisotropy Probe spacecraft (or WMAP) was launched in 2001. The main objective of the mission was precise measurement the of cosmic microwave background anisotropy. The product of the spacecraft are so-called full-sky maps, which may also be used for the galactic matter emission study. The mechanisms of galactic emission are known, and described theoretically, so a full-sky map can be built for every emission mechanism. One may expect that if all relevant mechanisms are included, the full-sky map in the specific wave band is equal to the sum of all galactic emission components, CMB radiation and radiation of the bright sources.

A component separation procedure was conducted in 2003 by D. Finkbeiner [1]. As a result, an excess of emission in the Galactic center region was found, which was called WMAP haze (after the confirmation by the Planck mission - WMAP/Planck haze). The radiation is assumed to be radially symmetric, extending to more than $20^{\circ}$.

The Fermi satellite with Large Area Telescope (LAT) apparatus was launched in 2008 in order to study full sky in gamma-ray band. A similar analysis may be conducted using the gamma-ray sky maps produced using Fermi-LAT event data. It was made in 2010 by M. Su et al. [2], and an excess of radiation was found. It was later called the Fermi bubbles - a symmetric radiation extending to $50^{\circ}$ above and below the Galactic Plane.

For now, there is no single explanation for the WMAP/Planck haze phenomenon. One class of theories assumes that several emission phenomena observed in the Galactic Center region have the same origin: WMAP/Planck haze, Fermi bubbles, soft X-ray emission and $2.3 \mathrm{GHz}$ radio-emission. This scenario has a characteristic property, which is a coincidence of spatial structures of all emission maps. The purpose of this paper is the examination of this hypothesis.

\footnotetext{
^e-mail: zhezher.yana@physics.msu.ru
} 


\section{Method}

Template fitting is a basic minimising scheme which was used in original WMAP analysis.

The initial map may be represented as:

$$
d_{i}=a_{j} P_{j i}+x_{i},
$$

where $a_{j}$ is an amplitude vector, $P_{j i}$ is template matrix and $x_{i}$ is an unknown component.

White noise makes a main contribution to noise maps of full-sky maps. It is statistically uncorrelated and it's covariation matrix is diagonal:

$$
\left\langle d_{i} d_{j}\right\rangle=N_{\underline{i}} \delta_{i j}
$$

where $N_{i}$ is a vector with length $N_{p i x}$.

The minimum of chi-squared criterion is obtained:

$$
a_{\alpha^{\prime}}=\left(P_{\alpha^{\prime} i} N_{i}^{-1} P_{\alpha i}\right)^{-1}\left(P_{\alpha i} N_{i}^{-1} d_{i}\right)
$$

Three interstellar medium emission mechanisms were considered:

- free-free emission - emission due to interaction between electrons and protons at the regions of partially ionised hydrogen $\left(H_{I I}\right) . H_{\alpha}$ emission (Lyman line transition of ionized hydrogen) is used as a template for this mechanism [3].

- synchrotron emission - an emission of electromagnetic waves by charged particles which propagate in a magnetic field with relativistic speed [4], [5].

- dust emission - emission of particles, including among their components silicon and carbon. An important dust property which allows to detect it is an ability to absorb and scatter light, it is called extinction [6].

One should notice, that the template for an unknown component should be also included in the template matrix $P_{j i}$, otherwise other components may compensate the part of studied emission. A traditional template for the WMAP/Planck haze is 2-D Gaussian ellipse with characteristic lengths $\sigma_{l}=15^{\circ}$ and $\sigma_{b}=20^{\circ}$.

Bright objects and the Galactic Plane are covered with a mask which covers $30.8 \%$ of the sky.

\section{Results}

Planck 2015 Low Frequency Instrument (LFI) full-sky maps were used as an initial data [7]. LFI creates maps on three different frequencies: 30, 44 and $70 \mathrm{GHz}$. The original WMAP analysis showed that the WMAP/Planck haze is the most intensive in at the frequencies $20-50 \mathrm{GHz}$, so 30 and $44 \mathrm{GHz}$ separated emission were used for the extended emission analysis.

According to extracted data,new component of extended emission is more intensive in northern hemisphere. But northern hemisphere is believed to be contaminated by unresolved sources..

The latest Fermi collaboration research [8] have shown the existence of substructures in the Fermi bubbles. The Planck data investigation doesn't indicate the existence of such substructures in the WMAP/Planck haze.

The size of the WMAP/Planck haze are approximated by two ellipses symmetric below and above the Galactic Plane. The derived values for symmetric case are $\sigma_{l}=\sigma_{b}=b_{0}=17.9^{\circ}$ in a good 


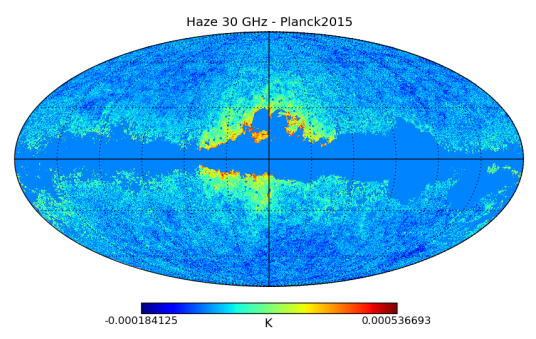

Figure 1. Extracted WMAP/Planck haze emission on $30 \mathrm{GHz}$.

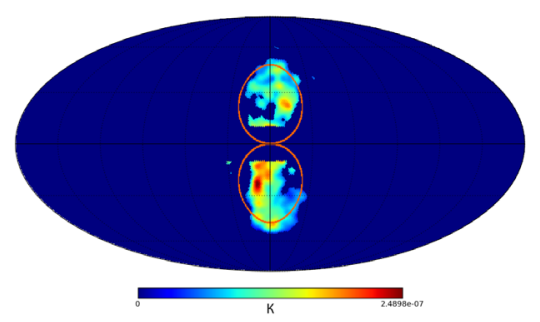

Figure 2. Comparison of the WMAP/Planck haze and Fermi bubbles sizes.

agreement with the Fermi bubbles sizes. For asymmetric case size estimations are: $\sigma_{l}=55.6^{\circ} \pm$ $0.6^{\circ}, \sigma_{b}=b_{0}=15.88^{\circ} \pm 0.18^{\circ}$.

The preliminary results for spectrum are derived. Taking the initial spectrum shape as $T_{v} \propto v^{\beta h}$, estimation for $\beta$ is $\beta=-2.86$, which is less than Planck results [9]: $\beta=-2.55 \pm 0.5$, and closer to the synchrotron scenario.

\section{Conclusion}

The indication on the similarity of spatial sizes of the WMAP/Planck haze and the Fermi bubbles is obtained. For the future work, it is proposed compare WMAP/Planck haze to other types of extended emission observed in the Galactic Center region and test other theoretical scenarios, which are dark matter and pulsar origin of WMAP/Planck haze.

\section{Acknowledgments}

The work is supported by the Russian Science Foundation grant 14-22-00161.

\section{References}

[1] D.P. Finkbeiner, ApJ 614 (2004) 186-193, astro-ph/0311547.

[2] M. Su, T. R. Slatyer, D. P. Finkbeiner, Astrophys.J. 724 1044-1082 (2010), astro-ph/1005.5480.

[3] D. P. Finkbeiner, Astrophys.J.Suppl 146407 (2003), astro-ph/0301558.

[4] C. G. T. Haslam et al., Astronomy and Astrophysics $1002209-219$ (1981).

[5] C. G. T. Haslam et al., Astronomy and Astrophysics Supplement Series 47 1, 2, 4-51, 53-142 (1982).

[6] D. P. Finkbeiner, M. Davis, D. J. Schegel, Astrophys.J. 524 867-886 (1999), astro-ph/9905128.

[7] J. A. Tauber et al., Astronomy\&Astrophysics 520 A1 (2010).

[8] Fermi-LAT Collaboration, Astrophys.J. 793134 (2014), astro-ph/1407.7905.

[9] P. A. R. Ade et al., astro-ph/1208.5483v1. 